Pacific Journal of Mathematics

COHOMOLOGY IN THE FINITE TOPOLOGY AND BRAVER 


\title{
COHOMOLOGY IN THE FINITE TOPOLOGY AND BRAUER GROUPS
}

\author{
RAYMOND T. HOOBLER
}

An exact sequence relating $\operatorname{Br}(X)$, the Brauer group of a regular scheme of dimension $\leqq 2$, and Amitsur cohomology (obtained as the cohomology of the sheaf of units on an appropriate Grothendieck topology) is derived by functorial methods. In order to do this we first show that any torsion element of $H^{1}\left(X_{e t}, \boldsymbol{G}_{m}\right)$, i.e., $\operatorname{Pic}(X)$, and $H^{2}\left(X_{e t}, \boldsymbol{G}_{m}\right)$, i.e., $\operatorname{Br}(X)$, is split by a finite, faithfully flat covering $Y \rightarrow X$. After proving a divisibility result for $\mathrm{Pic}(X)$ under such coverings and some preliminary investigation of cohomology in the topology defined from such coverings, the exact sequence which is analogous to that of Chase and Rosenberg is obtained.

Let $X$ be a regular scheme with $\operatorname{dim} X \leqq 2$, i.e. $\mathcal{O}_{y}$ is a regular local ring for all $y \in X$. Grothendieck has then shown that the Brauer group of the scheme $X, \operatorname{Br}(X)$, is isomorphic to $H^{2}\left(X_{e t}, G_{m}\right)$ where $X_{e t}$ is the etale site on $X$ [2]. On the other hand Chase and Rosenberg have given an exact sequence relating the kernel of $\mathrm{Br}(R) \rightarrow \mathrm{Br}(S)$ with $\breve{H}^{2}\left(S / R, G_{m}\right)$ where $S$ is a finite, faithfully flat $R$-algebra [5]. This result suggests that the Brauer group of $X, X$ a regular Japanese scheme with $\operatorname{dim} X \leqq 2$, might be described by $H^{2}\left(X_{f}, G_{m}\right)$ where $X_{f}$, the finite site on $X$, is the one suggested by using coverings of the type giving the Chase-Rosenberg exact sequence. Surprisingly, $H^{2}\left(X_{f}, \boldsymbol{G}_{m}\right)$ turns out to be too large. The measure of the difference lies in Pic $(X)$. If Pic $(X)$ is torsion, then $H^{2}\left(X_{f}, G_{m}\right)$ is the Brauer group of $X$.

Clearly we must first show that any Azumaya algebra on $X$ can be split by a finite, faithfully flat covering of $X$. This and some curious results on the behaviour of Pic $(X)$ constitute the major part of the first section. In the next section the cohomology groups, $H^{n}\left(X_{f}, G_{m}\right)$, are investigated by spectral sequence arguments, and a sequence similar to the Chase-Rosenberg sequence is derived. The result mentioned above then follows immediately from this sequence and the splitting theorems of the first section. In a forthcoming paper most of these results will be extended to the l-primary component of $\operatorname{Br}(X)$, $l$ a prime, for affine schemes $X$ of characteristic $l^{n}$. This accounts for the condition $\mathrm{Sp}(l)$ introduced in the second section.

We have generally adopted the style of Artin's Grothendieck Topologies [1] since it seems to be more readily available than SGAA [2]. This makes no difference in the results since all of the topologies 
we will use are generated from pretopologies and the Cech cohomology groups in such topologies can be computed as in Artin's notes [2; Exp. I, 2.1.4 and Exp. V, 2.1, d)]. A general knowledge of sheaves of Azumaya algebras on schemes is assumed. For details the reader can consult Grothendieck's Bourbaki talk [9] which is a straightforward extension of the work of Auslander and Goldman in the affine case [3]. We have adopted Bourbaki's convention of calling central, separable algebras (in the language of Auslander-Goldman) Azumaya algebras.

In what follows all rings and schemes are noetherian. All rings have 1 and are commutative unless they are Azumaya algebras.

1. Some splitting theorems. We will be interested in the fppf (faithfully flat of finite presentation), etale, Zariski, and finite topologies on (Sch), the category of schemes belonging to a fixed universe. They are generated from pretopologies [2; Exp. I, 2.1.2] where for $X \in(\mathrm{Sch})$, $\left\{X_{i} \stackrel{\varphi_{i}}{\longrightarrow} X\right\}_{i \in I}$ is a covering family of $X$ in the fppf, etale, Zariski, or finite pretopology if (1) for all $i \in I, \varphi_{i}$ is resp. flat and locally of finite presentation, resp. etale [6; Exp. 1], resp. an open immersion, resp. finite, flat and (2) $\bigcup_{i \in I} \varphi_{i}\left(X_{i}\right)=X$. Fixing a scheme $X$ we get the fppf, etale, Zariski, and finite sites on $X$ denoted by $X_{\text {fpp }}, X_{e t}$, $X_{\text {zar }}$, and $X_{f}$ respectively. They are formed by taking the full subcategory of (Sch)/ $X$ such that the structure map satisfies condition (1) on the covering families in the respective pretopologies. The set of covering families of $X$ will be denoted $\operatorname{Cov} X_{*}$ where $*=\mathrm{fppf}$, et, Zar, or $f$ respectively. These sites are related by morphisms of sites $X_{\mathrm{fppf}} \stackrel{\varepsilon_{2}}{\longrightarrow} X_{e t} \stackrel{\varepsilon_{1}}{\longrightarrow} X_{\mathrm{zar}}$ and $X_{\mathrm{fppf}} \stackrel{\tau}{\longrightarrow} X_{f}$ for any $X \in(\mathrm{Sch}) . \quad$ The category of sheaves of abelian groups on these sites will be denoted by $\widetilde{X}_{*}, *=$ fppf, et, Zar, or $f$.

Let $X$ be a scheme, $F$ a presheaf of abelian groups on the site $X_{*}$ where $*=$ fppf, et, Zar, or $f . \breve{H}^{n}\left(X_{*}, F\right)$ will denote the Cech cohomology of $F$ on $X_{*}$ and $H_{*}^{\check{n}}(F)$ will be the presheaf on $X_{*}$ given by $\breve{H}_{*}^{n}(F)(Y)=\check{H}^{n}(Y, F)$ for $Y \in \mathrm{Ob} X_{*}$. If $F \in \widetilde{X}_{*}, H^{n}\left(X_{*}, F\right)$ will denote the cohomology (by derived functors) of $F$ on the site $X_{*}$, and $\boldsymbol{H}_{*}^{n}(F)$ will be the presheaf on $X_{*}$ given by $\boldsymbol{H}_{*}^{n}(F)(Y)=H^{n}\left(Y_{*}\right.$, $F)$ for $Y \in \mathrm{Ob} X_{*}$. If $Y$ is a scheme over $X$, faithfully flat quasicompact descent theory shows that the functor of points of $Y$ defines a sheaf in any of the above topologies [6; Exp. VIII].

Lemma 1.1. Let $X$ be a scheme, $X=X_{1} \Perp \cdots \Perp X_{n}$ be a decomposition of $X$ into connected components, $i_{j}: X_{j} \rightarrow X, 1 \leqq j \leqq n$, be the inclusion map. Given $F_{j} \in \widetilde{X}_{j *}$, let $F=\bigoplus_{j=1}^{n} i_{j *} F_{j}$ where $* i$ s any of the above topologies. Then there is a natural isomorphism $\bigoplus_{j=1}^{n} H^{m}\left(X_{j *}, F_{j}\right) \rightarrow$ 
$H^{m}\left(X_{*}, F\right)$ for all $m$. Moreover if $F$ is representable, then $F=$ $\bigoplus_{j=1}^{n} i_{j *} i_{j}^{*} F$ in any of these topologies.

Proof. $X_{*}$ is noetherian with final object (of finite type in the language of [2]). Consequently $\oplus i_{j *} F_{j}$ defined as a presheaf is a sheaf and $\oplus H^{m}\left(X_{*}, i_{j *} F_{j}\right) \rightarrow H^{m}\left(X_{*}, F\right)$ is an isomorphism for all $m$ [1; II, 5]. Moreover for any $Y \stackrel{\varphi}{\longrightarrow} X, Y=\varphi^{-1}\left(X_{1}\right) \Perp \cdots \Perp \varphi^{-1}\left(X_{n}\right)$. Hence $X_{*}=X_{1 *} \times \cdots \times X_{n *}$ and so $i_{j *}$ is exact, $1 \leqq j \leqq n$. Thus the Leray spectral sequence for $i_{j}$ collapses, and $H^{m}\left(X_{j *}, F_{j}\right) \rightarrow H^{m}\left(X_{*}\right.$, $i_{j *} F_{j}$ ) is an isomorphism for all $m$.

Suppose $Y \stackrel{\varphi}{\longrightarrow} X$ represents $F$. Then $\varphi^{-1}\left(X_{j}\right)$ represents $i_{j}^{*} F$, and so $F=\bigoplus_{j=1}^{n} i_{j *} i_{j}^{*} F$.

Corollary 1.2. Let $X$ be a scheme, $X=X_{1} \Perp \cdots \Perp X_{n}$ be $a$ decomposition of $X$ into connected components, $\boldsymbol{G}_{m, X}$ be the sheaf of units on $X_{*}$. Then $\bigoplus_{j=1}^{n} H^{m}\left(X_{j *}, G_{m, X_{j}}\right) \rightarrow H^{m}\left(X_{*}, G_{m, X}\right)$ is an isomorphism for all $m$.

Recall that an integral domain $R$ is Japanese if the normalization of $R$ in any finite extension of its quotient field is an $R$-module of finite type $[7 ; 0,23.1]$. We extend this to schemes by calling a scheme $X$ Japanese if for every point $y \in X, \mathscr{O}_{y} / \boldsymbol{p}_{i}$ is Japanese for all $\boldsymbol{p}_{i} \in$ $\operatorname{Ass}\left(\mathscr{O}_{y}\right)$.

Proposition 1.3. Let $X$ be a regular, connected scheme with $\operatorname{dim} X \leqq 2$.

(1) If $X$ is Japanese, then $\left\{Y \rightarrow X \in \operatorname{Cov} X_{f} / Y\right.$ is normal and integral\} is a cofinal subset of $\operatorname{Cov} X_{f}$.

(2) Let $\left\{X_{i} \stackrel{\varphi_{i}}{\longrightarrow} X\right\}_{i \in I} \in \operatorname{Cov} X_{e t}$ be a finite set of etale maps with $X_{i}$ finite over the open subscheme $\varphi_{i}\left(X_{i}\right)$. Then there is $Y \rightarrow X \in \operatorname{Cov} X_{f}$ and $\left\{U_{i} \rightarrow Y\right\}_{i \in I} \in \operatorname{Cov} Y_{\text {zar }}$ which refines $\left\{X_{i} \times_{X} Y \rightarrow Y\right\}$.

Proof. (1) Let $Z \rightarrow X \in \operatorname{Cov} X_{f}, \bar{Z} \subset Z$ where $\bar{Z}$ is one of the irreducible components of $Z$ given the reduced subscheme structure. Let $Y \stackrel{\varphi}{\longrightarrow} X$ be the normalization of $X$ in the function field of $\bar{Z}$. Since $\bar{Z}$ is finite over $X, \varphi$ factors through $\bar{Z}$. Since $X$ is Japanese, $\varphi$ is finite and onto. To show that $\varphi$ is flat we may assume that $X=\operatorname{Spec} R, R$ a regular local ring with $\operatorname{dim} R \leqq 2$, and $Y=\operatorname{Spec} S$ where $S$ is the normalization of $R$ in a finite extension field of the quotient field of $R$. But then $S$ is a Cohen-Macauley ring since it is normal and $\operatorname{dim} S \leqq 2$. Hence $S$ is $R$-flat [7;0,17.3.5].

(2) Since $\varphi_{i}$ is etale $X_{i}$ is a regular scheme [6; Exp. I]. Moreover $V_{i}=\varphi_{i}\left(X_{i}\right)$ is an open set in $X$ since $\varphi_{i}$ is flat of finite presentation [8; 2.4.6]. Let $Y_{i}$ be the normalization of $X$ in the ring of regular 
functions on $X_{i}$. $\varphi_{i}^{\prime}: Y_{i} \rightarrow X$ is finite since the ring of regular functions on $X_{i}$ is a finite product of finite separable extension fields of the function field of $X . \quad \varphi_{i}^{\prime}$ is flat and onto by the argument above. Now $\varphi_{i}^{\prime-1}\left(V_{i}\right)=V_{i} \times Y_{i}$ is the normalization of $V_{i}$ in the ring of rational functions on $X_{i}$ where the fibred product is always over $X$. But $\varphi_{i}$ is finite over $V_{i}$. Hence $X_{i}$ is finite and normal over $V_{i}$ and so $X_{i} \cong$ $V_{i} \times Y_{i}$.

Let $Y=\mathrm{X}_{i \in I} Y_{i} \cdot \varphi: Y \rightarrow X \in \operatorname{Cov} X_{f}$ since $I$ is finite. Let $U_{i}=$ $\varphi^{-1}\left(V_{i}\right) \times Y$. Then the section $V_{i} \times Y_{i} \stackrel{\left(\cong, p_{2}\right)}{\longrightarrow} X_{i} \times Y_{i}$ induces a map $U_{i}=V_{i} \times Y \rightarrow X_{i} \times Y$ defined over $X$. Thus the Zariski covering $\left\{U_{i}\right\}$ of $Y$ refines the etale covering $\left\{X_{i} \times Y \rightarrow Y\right\}$.

Now suppose $X$ is a scheme with no embedded components, and let $y_{1}, \cdots, y_{n}$ be the generic points of the irreducible components of $X$. Then $\boldsymbol{R}_{X}$, the sheaf of rational functions in the Zariski topology, can be indentified with $\bigoplus_{j=1}^{n} i_{j *}\left(\mathscr{O}_{y_{j}}\right)$ where $i_{j}$ : Spec $\left(\mathscr{O}_{y_{j}}\right) \rightarrow X$ is the canonical map. Let $\boldsymbol{R}_{X}^{*}$ be the subsheaf of units of $\boldsymbol{R}_{X}$. There is an exact sequence of sheaves

$$
0 \rightarrow G_{m} \stackrel{k}{\longrightarrow} R_{X}^{*} \longrightarrow D_{i v_{X}} \longrightarrow 0
$$

where $\boldsymbol{G}_{m}$ is the sheaf of units and $\boldsymbol{D} \boldsymbol{D} \boldsymbol{v}_{X}=$ Cokernel $(k)$ is the sheaf of Cartier divisors on $X$ in the Zariski topology [12]. Since $\mathcal{O}_{y_{j}}^{*}$ is a constant sheaf on the irreducible space $Y=\operatorname{Spec}\left(\mathcal{O}_{y_{j}}\right), H^{1}\left(Y_{\mathrm{zar}}, \mathcal{O}_{y_{j}}^{*}\right)$ vanishes for $i>0$. In particular the long exact cohomology sequence for any open set $U \subseteq X$ give an exact sequence

$$
\text { (1.4) } 0 \rightarrow \Gamma\left(U, G_{m}\right) \rightarrow \Gamma\left(U, R_{X}^{*}\right) \rightarrow \Gamma\left(U, D_{i v_{X}}\right) \stackrel{\delta}{\longrightarrow} \operatorname{Pic}(U) \longrightarrow 0
$$

since $H^{1}\left(U_{\mathrm{zar}}, \boldsymbol{G}_{m}\right) \cong \operatorname{Pic}(U)$, the group of isomorphism classes of invertible $\mathscr{O}_{U}$-modules.

Theorem 1.5. Let $X$ be a regular, Japanese scheme with $\operatorname{dim} X \leqq$ 2, $U$ an open subscheme of $\bar{X}, \bar{X} \rightarrow X \in \operatorname{Cov} X_{f}$.

(1) If $y \in H^{1}\left(U_{e t}, G_{m}\right)$ is a torsion element, then there is $Y \rightarrow$ $X \in \operatorname{Cov} X_{f}$ and $\varphi: Y \rightarrow \bar{X} \in \operatorname{Mor} X_{f}$, such that $\varphi^{*}(y)=0$ in $H^{1}\left(\varphi^{-1}(U)_{e t}\right.$, $\left.\boldsymbol{G}_{m}\right)$.

(2) If $y \in H^{1}\left(U_{e t}, G_{m}\right)$ and $n$ is any positive integer, then there are $Y \rightarrow X \in \operatorname{Cov} X_{f}, \varphi: Y \rightarrow \bar{X} \in \operatorname{Mor} X_{f}$, and $\bar{y} \in H^{1}\left(\varphi^{-1}(U)_{e t}, G_{m}\right)$ such that $n \bar{y}=\varphi^{*}(y)$.

Proof. Since $H^{1}\left(Y_{e t}, G_{m}\right) \cong H^{1}\left(Y_{\mathrm{zar}}, G_{m}\right) \cong \operatorname{Pic}(Y)$ for any scheme 
$Y$ [2; Exp. IX, §3], (1) and (2) may be phrased in terms of Pic $(X)$, Pic $(Y)$, and Pic $(U)$. Moreover we may assume that $X$ and $\bar{X}$ are connected, $\bar{X}$ a normal, integral $X$-scheme.

(1) By (1.4) there is a Cartier divisor $D \in \Gamma\left(U, \operatorname{Div}_{\bar{x}}\right)$ such that $\delta(D)=y$ and $n D=(f), f \in \Gamma\left(U, \boldsymbol{R}_{X}^{*}\right)$, where $(f)$ denotes the Cartier divisor of the rational function $f . D$ is determined by a Zariski covering $\left\{U_{i}\right\}$ of $U$ and local equations $f_{i} \in \Gamma\left(U_{i}, \boldsymbol{R}_{X}^{*}\right)=K^{*}$ such that $f_{i} \cdot f_{j}^{-1} \in \Gamma\left(U_{i} \cap U_{j}, G_{m}\right)$ where $K^{*}$ is the group of units in the function field $K$ of $\bar{X}$. Moreover we may assume that $f^{-1} \cdot f_{i}^{n} \in \Gamma\left(U_{i}, G_{m}\right)$ for all $i$. Let $Y$ be the normalization of $X$ in $L=K(\sqrt[n]{f)}, \varphi: Y \rightarrow \bar{X}$, $V=\varphi^{-1}(U), V_{i}=\varphi^{-1}\left(U_{i}\right), g_{i}=\varphi^{*}\left(f_{i}\right) \in \Gamma\left(V_{i}, \boldsymbol{R}_{Y}^{*}\right)$ and $g=\varphi^{*}(f) \in \Gamma\left(V, \boldsymbol{R}_{Y}^{*}\right)$. Since $Y$ is integral, $\left\{V_{i}\right\}$ and $g_{i}$ define $\varphi^{*}(D) \in \Gamma\left(V, D_{i v_{Y}}\right)$. By construction there is $\bar{g} \in \Gamma\left(V, \boldsymbol{R}_{Y}^{*}\right)$ with $\bar{g}^{n}=g$. But then the Cartier divisor $\left(\bar{g}^{-1}\right)+\varphi^{*}(D)$ has local equations $\bar{g}_{i}=\bar{g}^{-1} \cdot g_{i}$ with $\bar{g}_{i}^{n} \in \Gamma\left(V_{i}, G_{m}\right)$. Since $Y$ is normal, $\bar{g}_{i} \in \Gamma\left(V_{i}, \mathscr{O}_{Y}\right)$, and so $\delta\left(\left(\bar{g}^{-1}\right)+\varphi^{*}(D)\right)=0=\varphi^{*}(\delta(D))=$ $\varphi^{*}(y)$. Finally the argument of Proposition 1.3 shows that $Y \rightarrow$ $X \in \operatorname{Cov} X_{f}$ as desired.

(2) Again we may assume that $\bar{X}$ is a normal, integral $X$ scheme. Represent $y$ by a Cartier divisor $D \in \Gamma\left(U\right.$, Div $\left._{\bar{X}}\right)$ where $D$ is defined by local equations $f_{i} \in \Gamma\left(U_{i}, R_{\bar{X}}^{*}\right)=K^{*},\left\{U_{i}\right\}, 1 \leqq i \leqq n$, a Zariski covering of $U$, such that $f_{i} \cdot f_{j}^{-1} \in \Gamma\left(U_{i} \cap U_{j}, G_{m}\right)$. Let $L=$ $K\left({ }^{n} / \overline{f_{i}}\right)_{i=1, \cdots, n}, Y$ be the normalization of $X$ in $L, \varphi: Y \rightarrow \bar{X}, V_{i}=$ $\varphi^{-1}\left(U_{i}\right)$, and $V=\varphi^{-1}(U)$. As in 1.3, $Y \rightarrow X \in \operatorname{Cov} X_{f}$. Moreover $\left(\sqrt[n]{f_{i}} \cdot \sqrt[n]{f_{j}^{-1}}\right)^{n} \in \Gamma\left(V_{i} \cap V_{j}, G_{m}\right)$ and so $\sqrt[n]{f_{i}} \cdot \sqrt[n]{f_{j}^{-1}} \in \Gamma\left(V_{i} \cap V_{j}, G_{m}\right)$ since $Y$ is normal. Thus $\left\{\sqrt[n]{f_{i}}\right\}$ are local equations of a Cartier divisor $E \in \Gamma\left(V, D_{i v_{Y}}\right)$. Clearly $n E=\varphi^{*}(D)$ and so $n \delta(E)=\delta\left(\varphi^{*}(D)\right)=\varphi^{*}(y)$ as desired.

The following result was pointed out by J. L. Verdier.

Proposition 1.6. Let $X$ be any scheme, $y$ a torsion element in $H^{1}\left(X_{e t}, \boldsymbol{G}_{m}\right)$. Then there is $Y \stackrel{\varphi}{\longrightarrow} X \in \operatorname{Cov} X_{f}$ such that $\phi^{*}(y)=0$ in $H^{1}\left(Y_{e t}, \boldsymbol{G}_{m}\right)$.

Proof. Let $L$ be the invertible $\mathscr{O}_{X}$-module whose class in Pic $(X)$ is $y, s \in \Gamma\left(X, L^{\otimes n}\right)$ the global section defining the isomorphism $\mathcal{O}_{X} \rightarrow L^{\otimes n}$. Then $R=\bigoplus_{j=0}^{\infty} L^{\otimes j} /(1-s)\left(\bigoplus_{j=0}^{\infty} L^{\otimes j}\right)$ is a coherent faithfully flat sheaf of $\mathscr{O}_{X}$-algebras, and clearly $L \Theta_{O_{X}} R \cong R$ as sheaves of $R$-modules. Let $Y=\operatorname{Spec}(R)$. Then $Y \stackrel{\varphi}{\longrightarrow} X \in \operatorname{Cov} X_{f}$ and $\varphi^{*}(L) \cong \mathcal{O}_{Y}$.

More surprising and much more interesting is the next splitting theorem for elements of $\mathrm{Br}(X)$, the Brauer group of $X$ [9]. 
THEOREM 1.7. Let $X$ be a regular Japanese scheme with $\operatorname{dim} X \leqq$ 2, $y \in H^{2}\left(X_{e t}, G_{m}\right)$. Then there is $\varphi: Y \rightarrow X \in \operatorname{Cov} X_{f}$ with $\varphi^{*}(y)=0$ in $H^{2}\left(Y_{e t}, \boldsymbol{G}_{m}\right)$.

Proof. Grothendieck has shown that $\operatorname{Br}(X) \subseteq H^{2}\left(Y_{e t}, G_{m}\right)$ and $\mathrm{Br}(X)$ is torsion for any scheme $X[9]$. Moreover if $X$ is regular with $\operatorname{dim} X \leqq 2$, then $\operatorname{Br}(X)=H^{2}\left(X_{e t}, G_{m}\right)$ [10]. Thus we may assume that $X$ is connected and $n \cdot y=0$ for some integer $n$. Since an Azumaya algebra can be split locally (in the Zariski topology) by finite etale coverings and $X$ is noetherian, we can find $\bar{X} \stackrel{\bar{\varphi}}{\longrightarrow} X$ and a finite Zariski covering $\left\{U_{i}\right\}$ of $\bar{X}$ such that $\left(\bar{\varphi} \mid U_{i}\right)^{*}(y)=0$ in $H^{2}\left(U_{i_{e t}}, \boldsymbol{G}_{m}\right)$ by Proposition 1.3. Thus it suffices to show that given $\bar{X} \rightarrow X \in$ $\operatorname{Cov} X_{f}, y \in H^{2}\left(\bar{X}_{e t}, G_{m}\right)$ an element of order $n$ and a Zariski covering $\left\{U_{i}\right\}$ of $\bar{X}$ such that $y \mid U_{i}=0$ in $H^{2}\left(U_{i_{e t}}, \boldsymbol{G}_{m}\right)$, then there is $Y \stackrel{\varphi}{\longrightarrow} \bar{X} \epsilon$ Mor $X_{f}, Y \rightarrow X \in \operatorname{Cov} X_{f}$, such that $\phi^{*}(y)=0$.

In the Leray spectral sequence for $\varepsilon_{1}: \bar{X}_{e t} \rightarrow \bar{X}_{\text {zar }}, R^{1} \varepsilon_{1 *}\left(G_{m}\right)=0$ since the Zariski topology contains enough coverings to split elements of Pic. Thus the exact sequence of low degree terms gives an exact sequence

$$
0 \longrightarrow H^{2}\left(\bar{X}_{\mathrm{Zar}}, \boldsymbol{G}_{m}\right) \stackrel{j}{\longrightarrow} H^{2}\left(\bar{X}_{e t}, \boldsymbol{G}_{m}\right) \longrightarrow \Gamma\left(\bar{X}, R^{2} \varepsilon_{1 *}\left(\boldsymbol{G}_{m}\right)\right) \text {. }
$$

Since $y$ is split by a Zariski covering of $\bar{X}$, there is an element $z \epsilon$ $H^{2}\left(\bar{X}_{\mathrm{zar}}, \boldsymbol{G}_{m}\right)$ of order $n$ with $j(z)=y$.

The spectral sequence $\check{H}^{p}\left(\bar{X}_{\mathrm{zar}}, \boldsymbol{H}_{\mathrm{zar}}^{q}\left(\boldsymbol{G}_{m}\right)\right) \Rightarrow H^{n}\left(\bar{X}_{\mathrm{zar}}, \boldsymbol{G}_{m}\right)$ gives an exact sequence

$$
0 \rightarrow \check{H}^{2}\left(\bar{X}_{\text {Zar }}, \boldsymbol{G}_{m}\right) \stackrel{i}{\longrightarrow} H^{2}\left(\bar{X}_{\text {zar }}, \boldsymbol{G}_{m}\right) \stackrel{\bar{\imath}}{\longrightarrow} \check{H}^{1}\left(\bar{X}_{\text {zar }}, \boldsymbol{H}_{\mathrm{Zar}}^{1}\left(\boldsymbol{G}_{m}\right)\right) \text { where }
$$
$\boldsymbol{H}_{\mathrm{zar}}^{1}\left(\boldsymbol{G}_{m}\right)=$ Pic. In particular $\bar{i}(y)$ may be represented by a Cech cocycle $\left\{y_{i j}\right\}, y_{i j} \in \operatorname{Pic}\left(U_{i} \cap U_{j}\right)$ with $y_{i j}+y_{j k}=y_{i k} \in \operatorname{Pic}\left(U_{i} \cap U_{j} \cap U_{k}\right)$, where $\left\{U_{i}\right\}$ is a finite Zariski covering of $\bar{X}$. Since $n \cdot \bar{i}(y)=0$, we may assume there are $y_{i} \in \operatorname{Pic}\left(U_{i}\right)$ with $y_{i}-y_{j}=n \cdot y_{i j} \in \operatorname{Pic}\left(U_{i} \cap U_{j}\right)$. By Theorem 1.5, there is $\bar{\varphi}: \bar{Y} \rightarrow \bar{X} \in \operatorname{Mor} X_{f}, \bar{Y} \rightarrow X \in \operatorname{Cov} X_{f}$ and $\bar{y}_{i} \in$ Pic $\left(\bar{\phi}^{-1}\left(U_{i}\right)\right)$ with $n \bar{y}_{i}=\bar{\phi}^{*}\left(y_{i}\right)$ for all $i$. Altering the Cech cocycle $\left\{\bar{\varphi}^{*}\left(y_{i j}\right)\right\} \in Z^{1}\left(\left\{\bar{\varphi}^{-1}\left(U_{i}\right)\right\}\right.$, Pic) by $\partial\left(\left\{\bar{y}_{i}^{-1}\right\}\right)$, it suffices to split $y$ in $H^{2}\left(\bar{X}_{\mathrm{zar}}, G_{m}\right)$ by $Y \rightarrow \bar{X}$ under the assumption that $n \cdot y_{i j}=0$ in Pic $\left(U_{i} \cap U_{j}\right)$ where $\left\{y_{i j}\right\}$ is a Cech cocycle representing $\bar{i}(y)$. Again by Theorem 1.5 there is $\bar{\varphi}: \bar{Y} \rightarrow \bar{X} \in \operatorname{Mor} X_{f}, \bar{Y} \rightarrow X \in \operatorname{Cov} X_{f}$, such that for each pair $i, j, \bar{\varphi}^{*}\left(y_{i j}\right)=0$ in $\operatorname{Pic}\left(\bar{\varphi}^{-1}\left(U_{i} \cap U_{j}\right)\right)$. Thus we may assume that $y=i(z), n \cdot z=0$ for some $z \in \check{H}^{2}\left(\bar{X}_{\mathrm{zar}}, \boldsymbol{G}_{m}\right)$. Moreover we may assume $\bar{X}$ is normal and integral by Proposition 1.3.

Represent $z$ by the Cech 2 cocycle $\left\{u_{i j_{k}}\right\}, u_{i j_{k}} \in \Gamma\left(V_{i} \cap V_{j} \cap V_{k}, \boldsymbol{G}_{m}\right)$ with $u_{i j k} u_{i j l}^{-1} u_{i k l} u_{j k l}^{-1}=1$ in $\Gamma\left(V_{i} \cap V_{j} \cap V_{k} \cap V_{l}, G_{m}\right)$ where $\left\{V_{i}\right\}_{i=1}^{n}$ is a finite covering of $\bar{X}$. Since $n z=0$, we may also assume that there are units $v_{i j} \in \Gamma\left(V_{i} \cap V_{i}, G_{m}\right)$ with $v_{i j} \cdot v_{i k}^{-1} \cdot v_{j k}=u_{i j k}^{n}$ for all $i, j, k$. Let 
$K$ be the function field of $\bar{X}, L=K\left(\sqrt[n]{v_{i j}}, \xi\right)_{1 \leqq i, j \leqq n}$ where $\xi$ is a primitive $m$ th root of unity, and $n=p^{r} m,(m, p)=1$, if $p=\operatorname{char} K>0$, $n=m$ otherwise. Let $Y$ be the normalization of $X$ in $L, \varphi: Y \rightarrow X$. The argument of Propositition 1.3 shows that $\phi \in \operatorname{Cov} X_{f}$ and, of course, there is a morphism $\psi: Y \rightarrow \bar{X}$ of $X$-schemes. Since $\sqrt[n]{v_{i j}} \in$ $\Gamma\left(\psi^{-1}\left(V_{i} \cap V_{j}\right), G_{m}\right)$ we may assume that the cohomology class $z \epsilon$ $\check{H}^{2}\left(Y_{\mathrm{zar}}, \boldsymbol{G}_{m}\right)$ that we must split can be represented by $\left\{v_{i j k}\right\}$ where $v_{i j k} \in$ $\Gamma\left(V_{i} \cap V_{j} \cap V_{k}, \mu_{n}\right)$ and $\mu_{n}$ is the sheaf of $n$th roots of unity. Now $Y$ has a global section of order $m$ where $n=p^{r} m,(m, p)=1$, if $p=$ char $L>0$ and $m=n$ otherwise. Hence $\mu_{n}$ is the constant sheaf $Z / m Z$ on $Y$ [6; Exp. XI, §6]. Since $Y$ is irreducible and a constant sheaf on an irreducible space is flask, $H^{2}\left(Y_{\text {zar }}, \mu_{n}\right)=0$. The Cech cohomology spectral sequence then shows that $\check{H}^{2}\left(Y_{\text {zar }}, \mu_{n}\right)=0$ and so $z=0$ in $\breve{H}^{2}\left(Y_{\text {zar }}, G_{m}\right)$ as desired.

Corollary 1.8. Let $A$ be an Azumaya algebra on $X, X$ a regular Japanese scheme with $\operatorname{dim} X \leqq 2$. Then there is $Y \stackrel{\varphi}{\longrightarrow} X \in \operatorname{Cov} X_{f}$ and a locally free coherent $\mathcal{O}_{Y}$-module $F$ such that $\varphi^{*}(A) \cong \operatorname{End}_{O_{Y}}(F)$.

REMARK. Let $X$ be a regular connected Japanese scheme with $\operatorname{dim} X \leqq 2$, and let $K$ be a finite extension of the field of rational functions on $X$. The Japanese assumption on $X$ was only used to show that the normalization of $X$ in $L$, a finite extension of $K$ obtained by adjoining $n$th roots of elements in $K$, was finite over $X$. Thus without the Japanese assumption Theorems 1.5 and 1.7 hold for $(n, p)=1$ where $p=\operatorname{char} K>0(n=$ order of $y$ in Theorem 1.5, (1) and Theorem (1.7)) since in this case the above extensions are separable and (2) of Proposition 1.3 did not use the Japanese assumption.

2. Finite cohomology. This section is devoted to determining the structure of $H^{n}\left(X_{f}, \boldsymbol{G}_{m}\right)$. The results when combined with the splitting theorems of the previous section describe the relationship between $\operatorname{Br}(X)$ and $H^{2}\left(X_{f}, G_{m}\right)$ for a regular Japanese scheme $X$ with $\operatorname{dim} X \leqq 2$.

Theorem 2.1. Let $X$ be a connected scheme, $Y \stackrel{\varphi}{\longrightarrow} X \in \operatorname{Cov} X_{f}$. Then there are natural maps $i_{*}: H^{n}\left(X_{*}, G_{m, X}\right) \rightarrow H^{n}\left(X_{*}, \varphi_{*} G_{m, Y}\right)$ and $N_{*}: H^{n}\left(X_{*}, \varphi_{*} G_{m, X}\right) \rightarrow H^{n}\left(X_{*}, G_{m, X}\right)$ for $n \geqq 0$ such that $N_{*} i_{*}$ is multiplication by $r k_{O X}\left(\varphi_{*} \mathscr{O}_{Y}\right)$ where $*=\mathrm{fppf}$, et, Zar, or $f$. Moreover $H^{n}\left(X_{f}, G_{m}\right)$ is a torsion group for all $n>0$.

Proof. Let $*=$ fppf. Then $i_{*}$ comes from the natural inclusion $\boldsymbol{G}_{m, X} \stackrel{i}{\longrightarrow} \varphi_{*} \boldsymbol{G}_{m, Y}$ where $\boldsymbol{G}_{m, X}, \varphi_{*} \boldsymbol{G}_{m, Y} \in \widetilde{X}_{\mathrm{fppp}}$. 
Let $R$ be a ring, $S$ a finite $R$-algebra which is free as an $R$-module. Define $N: S^{*} \rightarrow R^{*}$ where $R^{*}, S^{*}$ are the units of $R, S$ respectively by setting $N(u)=\operatorname{det}\left(L_{u}\right)$ where $L_{u}$ is the $R$-linear map of $S$ defined by left multiplication by the unit $u$. Since $L_{u}$ is an isomorphism, its determinant is a unit in $R$. The functorial properties of det show that $N$ is natural in $R$. Thus if $Y \stackrel{\varphi}{\longrightarrow} X$ is any finite faithfully flat morphism, then $\varphi_{*} \mathcal{O}_{Y}$ is a locally free coherent sheaf of $\mathscr{O}_{X}$-modules and $N$ extends to a morphism of sheaves $N: \varphi_{*}\left(\mathscr{O}_{Y}^{*}\right) \rightarrow \mathscr{O}_{X}^{*}[12$; Lecture 10] which is natural with respect to base change of $X$. Thus it extends to a map $N: \Phi_{*} G_{m, Y} \rightarrow G_{m, X} \in$ Mor $\widetilde{X}_{\mathrm{fppf}}$ by commutativity of the diagram below for any $X_{1} \rightarrow X_{2} \in$ Mor $X_{\text {fpp }}$ :

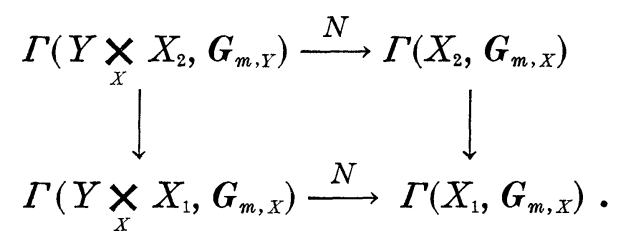

If $X$ is connected, then $\operatorname{rank}_{O_{X}}\left(\varphi_{*}\left(\mathcal{O}_{Y}\right)\right)$ is a constant and for any $X_{1} \rightarrow X_{i} \in \mathrm{Ob} X_{\mathrm{fppf}}$ the composite

$$
\Gamma\left(X_{1}, G_{m, X}\right) \stackrel{i}{\longrightarrow} \Gamma\left(Y \times X_{1}, G_{m, Y}\right) \stackrel{N}{\longrightarrow} \Gamma\left(X_{1}, G_{m, X}\right)
$$

sends $u$ to $u^{m}$ where $\left.m=\operatorname{rank}_{O_{X_{1}}}\left(\left(\varphi \times X_{1}\right)_{*}\left(\mathscr{O}_{Y \times X_{1}}\right)\right)=\operatorname{rank}_{C_{X}}\left(\varphi_{*} \mathscr{O}_{Y}\right)\right)$. Thus $N$ induces

$$
N_{*}: H^{n}\left(X_{\mathrm{fppf}}, \varphi_{*} \boldsymbol{G}_{m, Y}\right) \longrightarrow H^{n}\left(X_{\mathrm{fppf}}, \boldsymbol{G}_{m, X}\right)
$$

for all $n \geqq 0$ and by universality $N_{*} i_{*}$ is multiplication by $\operatorname{rank}_{\mathcal{O}_{X}}\left(\varphi_{*}\left(\mathscr{O}_{Y}\right)\right)$. The morphisms of sites $X_{\mathrm{fppf}} \rightarrow X_{*}$ where $*=$ et, Zar, or $f$ gives a $\operatorname{map} N: \varphi_{*} \boldsymbol{G}_{m, Y} \rightarrow \boldsymbol{G}_{m, X} \in \operatorname{Mor} \widetilde{X}_{*}$ which extends to $N_{*}: H^{n}\left(X_{*}, \varphi_{*} \boldsymbol{G}_{m Y}\right) \rightarrow$ $H^{n}\left(X_{*}, \boldsymbol{G}_{m_{X}}\right)$ with the desired properties. In particular the kernel of $i_{*}$ is torsion.

Finally we must show that $H^{n}\left(X_{f}, \boldsymbol{G}_{m}\right)$ is torsion for all $n>0$. If $Y \rightarrow X \in \operatorname{Cov} X_{f}$, then $\varphi^{*}: \widetilde{X}_{f} \rightarrow \widetilde{Y}_{f}$ is exact and left adjoint to $\varphi_{*}$. In particular

$$
H^{n}\left(Y_{f}, \boldsymbol{G}_{m, Y}\right) \longrightarrow H^{n}\left(Y_{f}, \boldsymbol{G}_{m, X}\right)
$$

is an isomorphism [1; II, 4.13] and so we will drop the subscript $X$ on $G_{m, X}$. We will use induction on $n, n \geqq 1$, to show that for a scheme $X, H^{n}\left(X_{f}, \boldsymbol{G}_{m}\right)$ is torsion. By Corollary 1.2 we may assume that $X$ is connected. Let $y \in H^{n}\left(X_{f}, G_{m}\right)$. There is $\varphi: Y \rightarrow X \in \operatorname{Cov} X_{f}$ such that $\varphi^{*}(y)=0$ in $H^{n}\left(Y_{f}, G_{m}\right)$ [1; II, 2.5], Now the map $\varphi^{*}$ may be written as the composite

$$
H^{n}\left(X_{f}, \boldsymbol{G}_{m, X}\right) \stackrel{i_{*}}{\longrightarrow} H^{n}\left(X_{f}, \varphi_{*} \boldsymbol{G}_{m, Y}\right) \stackrel{e_{n}}{\longrightarrow} H^{n}\left(Y_{f}, \boldsymbol{G}_{m, Y}\right)
$$


where $e_{n}$ is the edge homomorphism of the Leray spectral sequence for $\varphi: Y \rightarrow X, H^{p}\left(X_{f}, R^{q} \varphi_{*}\left(\boldsymbol{G}_{m, Y}\right)\right) \Rightarrow H^{n}\left(Y_{f}, \boldsymbol{G}_{m, Y}\right)$. By the above the kernel of $i_{*}$ is torsion and so it suffices to show that the kernel of $e_{n}$ is also torsion for $n \geqq 1$. For $n=1$, the exact sequence of low degree terms shows that $H^{1}\left(X_{f}, \varphi_{*} G_{m, Y}\right)$ is a subgroup of $H^{1}\left(Y_{f}, G_{m, Y}\right)$. For $n>1$, any element in the kernel of $e_{n}$ is in $d^{r}\left(E_{r}^{n-r, r-1}\right)$ for some $r$, $2 \leqq r \leqq n$. Hence it suffices to show that $H^{m}\left(X_{f}, R^{l} \mathscr{P}_{*}\left(G_{m}\right)\right)$ is torsion for $1 \leqq l \leqq n-1$ and $m \geqq 0$.

In general $R^{l} \Phi_{*}\left(\boldsymbol{G}_{m}\right)$ is the sheaf in $\widetilde{X}_{f}$ associated to the presheaf $\bar{X} \mapsto H^{l}\left(\bar{X} \times_{X} Y_{f}, G_{m, Y}\right)$ for any $\bar{X} \in \mathrm{Ob} X_{f}[1 ; I I, 4.7]$, and so is torsion by the induction hypothesis. Hence, it is sufficient to show that $H^{m}\left(X_{f}, F\right)$ is torsion for any torsion sheaf $F \in \widetilde{X}_{f}$ and $m \geqq 0$. Let ${ }_{n} F$ be the kernel of multiplication by $n$ on $F$. Then $F=\lim _{n} F$ and $H^{m}\left(X_{f}, F\right)=\lim H^{m}\left(X_{f},{ }_{n} F\right)$ since the topology on $X_{f}$ is $\overrightarrow{\text { noetherian }}$ [1, II, 5.3 and $\overrightarrow{5} .4]$. But multiplication by $n$ is the zero map on ${ }_{n} F$ and so by universality multiplication by $n$ on $H^{m}\left(X_{f},{ }_{n} F\right)$ is also the zero map for $m \geqq 0$. Since the limit of torsion sheaves is torsion, $H^{m}\left(X_{f}, F\right)$ is torsion for $m \geqq 0$.

Corollary 2.2. Let $X$ be a scheme, $Y \stackrel{\varphi}{\longrightarrow} X \in \operatorname{Cov} X_{f}$. Then the kernel of $H^{2}\left(X_{\mathrm{fpp}}, \boldsymbol{G}_{m}\right) \rightarrow H^{2}\left(Y_{\mathrm{fppf}}, \boldsymbol{G}_{m}\right)$ is torsion.

Proof. As in the proof above it is sufficient to show that the kernel of $H^{2}\left(X_{\mathrm{fppf}}, \varphi_{*} \boldsymbol{G}_{m}\right) \rightarrow H^{2}\left(Y_{\mathrm{fppf}}, \boldsymbol{G}_{m}\right)$ is torsion. But $\Gamma\left(X, R^{1} \varphi_{*} \boldsymbol{G}_{m}\right)=0$ since for any $\bar{X} \in \mathrm{Ob} X_{\mathrm{fppf}}$ and any element $y \in H^{1}\left(\bar{X} \times_{X} Y_{\mathrm{fppf}}, \boldsymbol{G}_{m}\right)=$ Pic $\left(\bar{X} \times_{X} Y\right)$ there is a Zariski covering $\left\{U_{i}\right\}$ of $\bar{X}$ such that $\left.y\right|_{U_{i} \times Y}=0$ in $H^{1}\left(U_{i} \times_{X} Y_{\mathrm{fppf}}, \boldsymbol{G}_{m}\right)$ [12; Lecture 10]. Hence this map is injective.

We are now in a position to evaluate some of the cohomology groups of $G_{m}$ in the finite topology. If $G$ is a group or a presheaf on $X_{*}$ in some topology, let $G_{t}$ and $G(l)$ denote the subgroup or subpresheaf consisting of torsion elements and elements whose order is a power of $l$ respectively. For a fixed scheme $X$ we have morphisms of sites $X_{\mathrm{fppf}} \stackrel{\tau}{\longrightarrow} X_{f}$ and $X_{\mathrm{fppf}} \stackrel{\varepsilon_{2}}{\longrightarrow} X_{e t}$. Grothendieck has shown that $\varepsilon_{2}$ induces an isomorphism $H^{n}\left(X_{e t}, G_{m}\right) \rightarrow H^{n}\left(X_{\text {fpp }}, G_{m}\right)$ for all $n \geqq 0$ [11; Appendix]. This immediately extends the results of the previous section to equivalent results about $H^{i}\left(X_{\text {fppr }}, G_{m}\right), i=0,1,2$.

The proof of the main theorem is based on the Kummer sequence

$$
0 \longrightarrow \mu_{n} \longrightarrow G_{m} \stackrel{n}{\longrightarrow} G_{m} \longrightarrow 0
$$

where $n$ is the $n$th power map. Since taking $n$th roots of global units gives a finite faithfully flat extension, this is an exact sequence of sheaves in both $\widetilde{X}_{f}$ and $\widetilde{X}_{\mathrm{fppr}}$. We will leave it to the context to 
determine in which topology $\mu_{n}$ and $\boldsymbol{G}_{m}$ are sheaves.

Definition. A scheme $X$ satisfies $\operatorname{Sp}(l)$ for some prime $l$ if for any $Y \in \mathrm{ObX}_{f}$ and $y \in H^{1}\left(Y_{\mathrm{fppf}}, \boldsymbol{G}_{m}\right)$, there is $\bar{Y} \in \mathrm{Ob} X_{f}, \bar{Y} \stackrel{\varphi}{\longrightarrow} Y \in \operatorname{Mor} X_{f}$ and $\bar{y} \in H^{1}\left(\bar{Y}_{\mathrm{fpp}}, \boldsymbol{G}_{m}\right)$ with $l \bar{y}=\varphi^{*}(y)$.

By Theorem 1.5 if $X$ is a regular Japanese scheme with $\operatorname{dim} X \leqq$ 2 , then $X$ satisfies $\operatorname{Sp}(l)$ for all primes $l$.

Theorem 2.4. Let $X$ be any scheme. Then $H^{1}\left(X_{f}, G_{m}\right) \cong \operatorname{Pic}_{t}(X)$, and $R^{1} \tau_{*}\left(G_{m}\right)$ is torsion free. There are exact sequences

$$
\begin{aligned}
0 \rightarrow \operatorname{Pic}_{t}(X) & \rightarrow \operatorname{Pic}(X) \rightarrow \Gamma\left(X, R^{1} \tau_{*}\left(\boldsymbol{G}_{m}\right)\right) \rightarrow \\
H^{2}\left(X_{f}, G_{m}\right) & \stackrel{\tau *}{\longrightarrow} F^{1} H^{2}\left(X_{\mathrm{fpp}}, G_{m}\right) \rightarrow H^{1}\left(X_{f}, R^{1} \tau_{*} G_{m}\right) \text { and } \\
0 & \rightarrow F^{1} H^{2}\left(X_{\mathrm{fpp}}, \boldsymbol{G}_{m}\right) \rightarrow H^{2}\left(X_{\mathrm{fppf}}, \boldsymbol{G}_{m}\right) \rightarrow \Gamma\left(X_{f}, R^{2} \tau_{*} \boldsymbol{G}_{m}\right),
\end{aligned}
$$

where $F^{1} H^{2}\left(X_{\mathrm{fpp}}, \boldsymbol{G}_{m}\right)$ is a torsion group. If Pic $(X)$ is torsion, then $\tau_{*}$ is injective. If $X$ satisfies $\operatorname{Sp}(l)$, then $\Gamma\left(X, R^{1} \tau_{*}\left(G_{m}\right)\right)$ is l-divisible and

$$
\tau_{*}(l): H^{2}\left(X_{f}, \boldsymbol{G}_{m}\right)(l) \longrightarrow\left(F^{1} H^{2}\left(X_{\mathrm{fppr}}, \boldsymbol{G}_{m}\right)\right)(l)
$$

is onto. If $\operatorname{Pic}(X)$ is torsion, then $\tau_{*}(l)$ is an isomorphism.

Proof. For any $Y \rightarrow X \in \mathrm{Ob} X_{f}$, the spectral sequence coming from $\tau: X_{\text {fppf }} \rightarrow X_{f}$ applied to the Kummer sequence gives a large diagram with exact columns coming from the low degree terms of the spectral sequences and all but the third row exact from the Kummer sequence:

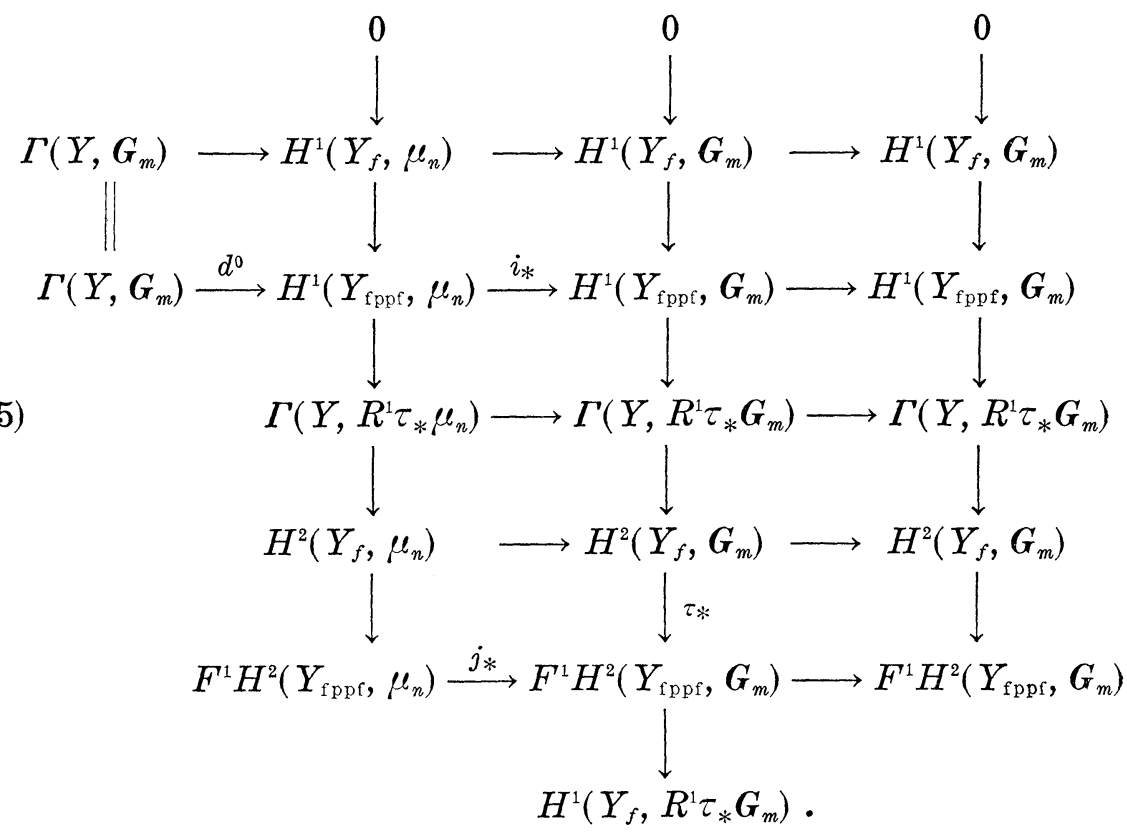


The middle column suitably interpreted is the 6 term exact sequence of the theorem. By definition of the filtration on the spectral sequence,

$$
0 \longrightarrow F^{1} H^{2}\left(Y_{\mathrm{fppf}}, \boldsymbol{G}_{m}\right) \longrightarrow H^{2}\left(Y_{\mathrm{fppf}}, \boldsymbol{G}_{m}\right) \longrightarrow E_{\infty}^{2,0} \sqsubseteq \Gamma\left(Y_{f}, R^{2} \tau_{*} G_{m}\right)
$$

is exact. By Corollary 2.2, $F^{1} H^{2}\left(Y_{\mathrm{fppf}}, G\right)$, which consists of these elements in $H^{2}\left(Y_{\mathrm{fppf}}, \boldsymbol{G}_{m}\right)$ that are split by $\bar{Y} \rightarrow Y \in \operatorname{Cov} Y_{f}$ for some $\bar{Y}$, is a torsion group. Moreover $H^{1}\left(Y_{\mathrm{fpp}}, \boldsymbol{G}_{m}\right) \cong \operatorname{Pic}(Y)$. Thus the naturality in $Y$ of (2.5), Proposition 1.6, and Theorem 2.1 combine to show that $H^{1}\left(X_{f}, G_{m}\right) \cong \operatorname{Pic}_{t}(X)$ for any scheme $X$.

In order to show that $R^{1} \tau_{*}\left(G_{m}\right)$ is torsion free, it is sufficient to show that $R^{1} \tau_{*}\left(\mu_{n}\right)$, the sheaf associated to $Y \rightarrow H^{1}\left(Y_{\mathrm{fppf}}, \mu_{n}\right)$ for $Y \rightarrow$ $X \in \mathrm{Ob} X_{f}$, is 0 [1; II 4.7]. So given $Y \rightarrow X \in \mathrm{Ob} X_{f}$ and $y \in H^{1}\left(Y_{\mathrm{fppf}}, \mu_{n}\right)$, we have $n \cdot i_{*}(y)=0$. By Proposition 1.6, there is $\bar{Y} \stackrel{\varphi}{\longrightarrow} Y \in \operatorname{Cov} Y_{f}$ with $\varphi^{*}\left(i_{*}(y)\right)=0$. Hence $\varphi^{*}(y)=d^{\circ}(u)$ for some $u \in \Gamma\left(\bar{Y}, \boldsymbol{G}_{m}\right)$. Adjoining an $n$th root of $u$ to $\bar{Y}$, we get $\widetilde{Y} \stackrel{\widetilde{\varphi}}{\longrightarrow} Y \in \operatorname{Cov} Y_{f}$ such that $\widetilde{\varphi}^{*}(y)=0$ in $H^{1}\left(\widetilde{Y}_{\mathrm{fppf}}, \mu_{n}\right)$ by the exact cohomology sequence coming from (2.3). Since there is a covering map of $Y$ which splits $y$, the associated sheaf is trivial. In particular if Pic $X$ is torsion, then $H^{2}\left(X_{f}, G_{m}\right)$ contains a torsion free subgroup which contradicts 2.1 unless $\Gamma\left(X, R^{1} \tau_{*}\left(G_{m}\right)\right)=0$.

Now suppose $X$ satisfies $\operatorname{Sp}(l)$. If $\Gamma\left(X, R^{2} \tau_{*}\left(\mu_{l}\right)\right) \rightarrow \Gamma\left(X, R^{2} \tau_{*}\left(G_{m}\right)\right)$ is injective, then $\Gamma\left(X, R^{1} \tau_{*}\left(G_{m}\right)\right) \rightarrow \Gamma\left(X, R^{1} \tau_{*}\left(G_{m}\right)\right)$ is onto. Thus it is sufficient to show that for any $Y \rightarrow X \in \operatorname{Cov} X_{f}, y \in H^{2}\left(Y_{\text {fppf }}, \mu_{l}\right)$ such that $j_{*}(y)=0$ in $H^{2}\left(Y_{\mathrm{f} p p \mathrm{f}}, \boldsymbol{G}_{m}\right)$, there is $\bar{Y} \rightarrow X \in \operatorname{Cov} X_{f}$ and $\bar{Y} \stackrel{\varphi}{\longrightarrow} Y \in$ Mor $X_{f}$ such that $\varphi^{*}(y)=0$. Since $j_{*}(y)=0$, there is $z \in \operatorname{Pic}(Y)$ such that $d^{1}(z)=y$ where $d^{1}: H^{1}\left(Y_{\mathrm{fppf}}, G_{m}\right) \rightarrow H^{2}\left(Y_{\mathrm{fppf}}, \mu_{l}\right)$ is the connecting homomorphism coming from (2.3). Since $X$ satisfies $\operatorname{Sp}(l)$, there is $\bar{Y} \rightarrow$ $X \in \operatorname{Cov} X_{f}, \bar{Y} \stackrel{\varphi}{\longrightarrow} Y \in \operatorname{Mor} X_{f}$, and $\bar{z} \in \operatorname{Pic}(\bar{Y})$ such that $l \cdot \bar{z}=\varphi^{*}(z)$. But then $\varphi^{*}(y)=0$ since $d^{1}(l \cdot \bar{z})=\varphi^{*}(y)=0$.

Finally we must show that

$$
\tau_{*}(l): H^{2}\left(X_{f}, G_{m}\right)(l) \rightarrow\left(F^{1} H^{2}\left(X_{\mathrm{fpp}}, G_{m}\right)\right)(l)
$$

is onto. First note that for any presheaf of sets $F$ on $X_{f}, U \in \mathrm{Ob} X_{f}$, and any element $y \in \boldsymbol{H}_{f}^{\circ}(F)(U)$, there is a covering $V \stackrel{\varphi}{\longrightarrow} U$ and an element $y_{1} \in F(V)$ which represents $\varphi^{*}(y)$ in $\boldsymbol{H}_{f}^{0}(F)(V)$. This may be seen by representing $y$ by an element $y_{1} \in F(V)$ such that $p_{1}^{*}\left(y_{1}\right)=$ $p_{2}^{*}\left(y_{1}\right) \in F\left(V \times_{U} V\right)$ where $V \stackrel{\varphi}{\longrightarrow} U$ is a covering of $U$ and $p_{i}$ is the projection map onto the $i$ th factor. Then $\varphi^{*}(y)$ is represented by $p_{1}^{*}\left(y_{1}\right) \in$ $F(V \times V)$ where $V \times_{U} V \stackrel{p_{2}}{\longrightarrow} V$ is a covering of $V$. Since $p_{1}^{*}\left(y_{1}\right)=p_{2}^{*}\left(y_{1}\right)$, $y_{1} \in F(V)$ represents $\varphi^{*}(y)$.

Now if $H^{1}\left(X_{f}, R^{1} \tau_{*} G_{m}\right)(l)=0$, then the exact long middle column of (2.5) shows that $\tau_{*}(l)$ is onto. So suppose $x \in H^{1}\left(X_{f}, R^{1} \tau_{*} G_{m}\right)$ and $l \cdot x=$ 
0 . Since $H^{1}\left(X_{f}, R^{1} \tau_{*} G_{m}\right) \cong \check{H}^{1}\left(X_{f}, R^{1} \tau_{*} G_{m}\right)$, there is $Y \rightarrow X \in \operatorname{Cov} X_{f}$ and $y \in \Gamma\left(Y \times_{X} Y, R^{1} \tau_{*} G_{m}\right)$ satisfying the cocycle identity which represents $x$ in $H^{1}\left(X_{f}, R^{1} \tau_{*} G_{m}\right)$. Moreover we may choose $Y$ so that for some element $\bar{y} \in \Gamma\left(Y, R^{1} \tau_{*} G_{m}\right)$ we have $p_{1}^{*}(\bar{y})-p_{2}^{*}(\bar{y})=l y$. Since $R^{1} \tau_{*} \boldsymbol{G}_{m}$ is the sheaf in $\widetilde{X}_{f}$ coming from the presheaf $H_{f p p r}^{1}\left(\boldsymbol{G}_{m}\right)$, the above remark and the observation that $\boldsymbol{H}^{0}\left(\boldsymbol{H}^{0}(F)\right)$ is the sheaf associated to $F$ shows that there is $Y_{1} \stackrel{\varphi}{\longrightarrow} Y \in \operatorname{Cov} Y_{f}$ and $\bar{z} \in H^{1}\left(Y_{1_{\mathrm{fppr}}}, G_{m}\right)=$ Pic $\left(Y_{1}\right)$ such that $\bar{z}$ represents $\varphi^{*}(\bar{y})$. Thus since $X$ satisfies $\operatorname{Sp}(l)$, we may assume that there is $\bar{y}_{1} \in \Gamma\left(Y, R^{1} \tau_{*} G_{m}\right)$ with $l \bar{y}_{1}=\bar{y}$ by taking a refinement in $\operatorname{Cov} X_{f}$ of $Y \rightarrow X$ if necessary. Altering the original Cech cocycle $y$ by the boundary $p_{2}^{*}\left(\bar{y}_{1}\right)-p_{1}^{*}\left(\bar{y}_{1}\right)$ and denoting the resulting cocycle by $z_{1} \in \Gamma\left(Y \times_{X} Y, R^{1} \tau_{*} G_{m}\right)$, we find that $l z_{1}=0$. Since $R^{1} \tau_{*} G_{m}$ is torsion free, $z_{1}=0$, and so $y=0$.

COROLlaRY 2.5. Let $X$ be a regular Japanese scheme with $\operatorname{dim} X \leqq$ 2. Then there is an exact sequence

$$
\begin{aligned}
0 \rightarrow \operatorname{Pic}_{t}(X) \longrightarrow \operatorname{Pic}(X) & \longrightarrow \Gamma\left(X_{f}, R^{1} \tau_{*} G_{m}\right) \\
& \longrightarrow H^{2}\left(X_{f}, G_{m}\right) \longrightarrow \operatorname{Br}(X) \longrightarrow 0
\end{aligned}
$$

where $\operatorname{Br}(X)$ is the Brauer group of $X$ and $\Gamma\left(X_{f}, R^{1} \tau_{*} G_{m}\right)$ is a vector space over the rationals of dimension $=\operatorname{dim}_{Q}\left(\operatorname{Pic}(X) \boldsymbol{\bigotimes}_{Z} Q\right)$. In particular if $\operatorname{Pic}_{t}(X)=\operatorname{Pic}(X)$, then $H^{2}\left(X_{f}, G_{m}\right) \cong \operatorname{Br}(X)$.

Proof. By Theorem 1.7 every element of $H^{2}\left(X_{\mathrm{fppr}}, \boldsymbol{G}_{m}\right)$ can be split by a covering map of $X$ in $X_{f}$. Hence

$$
F^{1} H^{2}\left(X_{\mathrm{fpp}}, G_{m}\right)=H^{2}\left(X_{\mathrm{fppr}}, G_{m}\right),
$$

and by Grothendieck's result this is $\operatorname{Br}(X)$. The dimension statement follows immediately since the other terms are torsion groups.

COROLLARY 2.6 Let $X$ be a regular Japanese scheme with $\operatorname{dim} X \leqq$ 2. Then $H^{2}\left(X_{\mathrm{fppr}}, \boldsymbol{G}_{m}\right) \cong \check{H}^{2}\left(X_{\mathrm{fppr}}, \boldsymbol{G}_{m}\right)$.

Proof. The morphism $\tau: X_{\text {fppf }} \rightarrow X_{f}$ induces a mapping of spectral sequences between Cech and sheaf cohomology:

$$
\begin{aligned}
{\left[\check{H}^{p}\left(X_{f}, \boldsymbol{H}_{f}^{q}\left(\boldsymbol{G}_{m}\right)\right)\right.} & \left.\Longrightarrow H^{n}\left(X_{f}, \boldsymbol{G}_{m}\right)\right] \longrightarrow\left[\check{H}^{p}\left(X_{\mathrm{fppf}}, \boldsymbol{H}_{\mathrm{fppf}}^{q},\left(\boldsymbol{G}_{m}\right)\right)\right. \\
& \left.\Longrightarrow H^{n}\left(X_{\mathrm{fppf}}, \boldsymbol{G}_{m}\right)\right] .
\end{aligned}
$$

The mapping between exact sequences of low degree terms gives

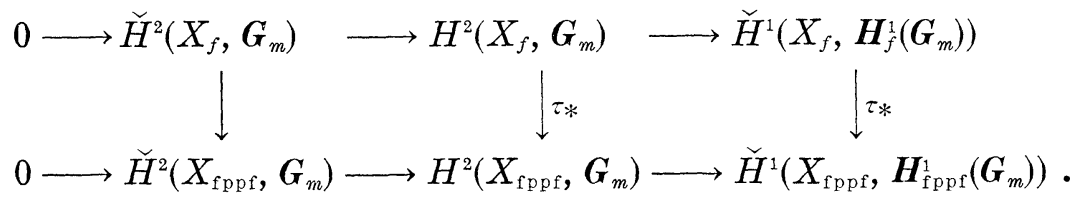


Moreover $\boldsymbol{H}_{f}^{1}\left(\boldsymbol{G}_{m}\right)(Y)=\operatorname{Pic}_{t}(Y)$. If $x \in \check{H}^{1}\left(X_{f}, \boldsymbol{H}_{f}^{1}\left(\boldsymbol{G}_{m}\right)\right)$, then it can be represented by $y \in \operatorname{Pic}_{t}\left(Y \times_{X} Y\right)$ where $Y \rightarrow X \in \operatorname{Cov} X_{f}$. Since $Y \times_{X} Y$ is finite over $X, y$ can be split by a Zariski covering of $X$ [12]. Thus $\bar{\tau}_{*}(x)=0$ and so $\tau_{*}$ factors through $\breve{H}^{2}\left(X_{\mathrm{fppf}}, \boldsymbol{G}_{m}\right)$. Since $\tau_{*}$ is surjective, we get the desired conclusion.

REMARK. The argument Bass uses to prove that $K^{0}(R)$ is a finitely generated abelian group for $R$ a finite $Z$-algebra [4; Theorem 18.6] may be copied to show that Pic $(R)$ is a finite group if $R$ is a finite $Z$-algebra.

\section{REFERENCES}

1. M. Artin, Grothendieck Topologies, Mimeographed Notes, Harvard University, 1962.

2. M. Artin and A. Grothendieck. Seminaire de Geometrie Algebrique, Cohomologie Etale des Schemas, Exposes I-XIV, Institut des Hautes Etudes Scientifique, 1963-64.

3. M. Auslander and O. Goldman, The Brauer group of a commutative ring, Trans. Amer. Math. Soc., 97 (1960), 367-409.

4. H. Bass, K-Theory and Stable Algebra, Publications Mathematique, Institut des Hautes Etudes Scientifique, no. 22, pp. 5-60.

5. S. Chase and A. Rosenberg, Amitsur Cohomology and the Brauer Group, Memoirs Amer. Math. Soc., no. 52 (1964), 34-79.

6. A. Grothendieck, Seminaire de Geometrie Algebrique, Exposes I-XI, Institut des Hautes Etudes Scientifique, 1960-61.

7. —_ Elements de Geometrie Algebrique, Chap. IV, part 1, Publications Mathematiques, Institute des Hautes Etudes Scientifique, no. 20.

8. ㄴ. Elements de Geometrie Algebrique, Chap. IV, part 2, Publications Mathematiques, Institut des Hautes Etudes Scientifique, no. 24.

9. - Le Groupe de Brauer, I, Dix Exposes sur la Cohomologie des Schemas, North-Holland Pub. Co., Amsterdam, (1969), 46-65.

10. L Le Groupe de Brauer, II, Dix Exposes sur la Cohomologie des Schemas, North-Holland Pub. Co., Amsterdam, (1969), 66-87.

11. - Le Groupe de Brauer, III, Dix Exposes sur la Cohomologie des Schemas, North-Holland Pub. Co., Amsterdam, (1969), 88-188.

12. D. Mumford, Lectures on Curves on an Algebraic Surface, Annals of Mathematics, no. 59, Princeton Universitity Press (1966).

Received April 2, 1971 and in revised form March 24, 1972. The preparation of this paper was partially supported by NSF Grant GP-8718. This work is an extension of some of the ideas in my thesis. I would like to thank G. P. Hochschild, my thesis advisor, for his patience and constant encouragement. I would also like to thank J. L. Verdier and H. Bass for their comments and suggestions during the preparation of this paper.

COLUMBIA UNIVERSITY

AND

Graduate Center, City University of New York 



\section{PACIFIC JOURNAL OF MATHEMATICS}

\section{EDITORS}

\section{H. SAMELSON}

Stanford University

Stanford, California 94305

\section{R. HOBBY}

University of Washington

Seattle, Washington 98105

\section{J. DugundjI}

Department of Mathematics University of Southern California Los Angeles, California 90007

RICHARD ARENS

University of California

Los Angeles, California 90024

\section{ASSOCIATE EDITORS}
E. F. BECKENBACH
B. H. NeumanN
F. WOLF
K. YOSHIDA

\section{SUPPORTING INSTITUTIONS}

\author{
UNIVERSITY OF BRITISH COLUMBIA \\ CALIFORNIA INSTITUTE OF TECHNOLOGY \\ UNIVERSITY OF CALIFORNIA \\ MONTANA STATE UNIVERSITY \\ UNIVERSITY OF NEVADA \\ NEW MEXICO STATE UNIVERSITY \\ OREGON STATE UNIVERSITY \\ UNIVERSITY OF OREGON \\ OSAKA UNIVERSITY
}

\author{
UNIVERSITY OF SOUTHERN CALIFORNIA \\ STANFORD UNIVERSITY \\ UNIVERSITY OF TOKYO \\ UNIVERSITY OF UTAH \\ WASHINGTON STATE UNIVERSITY \\ UNIVERSITY OF WASHINGTON \\ AMERICAN MATHEMATICAL SOCIETY \\ NAVAL WEAPONS CENTER
}

The Supporting Institutions listed above contribute to the cost of publication of this Journal, but they are not owners or publishers and have no responsibility for its content or policies.

Mathematical papers intended for publication in the Pacific Journal of Mathematics should be in typed form or offset-reproduced, (not dittoed), double spaced with large margins. Underline Greek letters in red, German in green, and script in blue. The first paragraph or two must be capable of being used separately as a synopsis of the entire paper. The editorial "we" must not be used in the synopsis, and items of the bibliography should not be cited there unless absolutely necessary, in which case they must be identified by author and Journal, rather than by item number. Manuscripts, in duplicate if possible, may be sent to any one of the four editors. Please classify according to the scheme of Math. Rev. Index to Vol. 39. All other communications to the editors should be addressed to the managing editor, Richard Arens, University of California, Los Angeles, California, 90024.

50 reprints are provided free for each article; additional copies may be obtained at cost in multiples of 50 .

The Pacific Journal of Mathematics is published monthly. Effective with Volume 16 the price per volume (3 numbers) is $\$ 8.00$; single issues, $\$ 3.00$. Special price for current issues to individual faculty members of supporting institutions and to individual members of the American Mathematical Society: $\$ 4.00$ per volume; single issues $\$ 1.50$. Back numbers are available.

Subscriptions, orders for back numbers, and changes of address should be sent to Pacific Journal of Mathematics, 103 Highland Boulevard, Berkeley, California, 94708.

PUBLISHED BY PACIFIC JOURNAL OF MATHEMATICS, A NON-PROFIT CORPORATION

Printed at Kokusai Bunken Insatsusha (International Academic Printing Co., Ltd.), 270, 3-chome Totsuka-cho, Shinjuku-ku, Tokyo 160, Japan. 


\section{Pacific Journal of Mathematics}

\section{Vol. 42, No. $3 \quad$ March, 1972}

Catherine Bandle, Extensions of an inequality by Pólya and Schiffer for vibrating membranes ................................ 543

S. J. Bernau, Topologies on structure spaces of lattice groups.......... 557

Woodrow Wilson Bledsoe and Charles Edward Wilks, On Borel product measures .......................................

Eggert Briem and Murali Rao, Normpreserving extensions in subspaces of

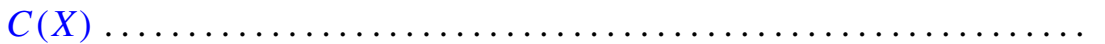

Alan Seymour Cover, Generalized continuation.................. 589

Larry Jean Cummings, Transformations of symmetric tensors .......... 603

Peter Michael Curran, Cohomology of finitely presented groups .......... 615

James B. Derr and N. P. Mukherjee, Generalized quasicenter and

hyperquasicenter of a finite group ...................... 621

Erik Maurice Ellentuck, Universal cosimple isols .................. 629

Benny Dan Evans, Boundary respecting maps of 3-mainfolds .......... 639

David F. Fraser, A probabilistic method for the rate of convergence to the

Dirichlet problem .................................. 657

Raymond Taylor Hoobler, Cohomology in the finite topology and Brauer

groups ..................................... 667

Louis Roberts Hunt, Locally holomorphic sets and the Levi form ........ 681

B. T. Y. Kwee, On absolute de la Vallée Poussin summability............ 689

Gérard Lallement, On nilpotency and residual finiteness in semigroups .... 693

George Edward Lang, Evaluation subgroups of factor spaces........... 701

Andy R. Magid, A separably closed ring with nonzero torsion pic ....... 711

Billy E. Rhoades, Commutants of some Hausdorff matrices ............. 715

Maxwell Alexander Rosenlicht, Canonical forms for local derivations . . . . 721

Cedric Felix Schubert, On a conjecture of L. B. Page ................ 733

Reinhard Schultz, Composition constructions on diffeomorphisms of $S^{p} \times S^{q}$

J. P. Singhal and H. M. (Hari Mohan) Srivastava, A class of bilateral generating functions for certain classical polynomials ....

Richard Alan Slocum, Using brick partitionings to establish conditions which insure that a Peano continuum is a 2-cell, a 2-sphere or an annulus...

James F. Smith, The p-classes of an $H^{*}$-algebra ...

Jack Williamson, Meromorphic functions with negative zeros and positive

poles and a theorem of Teichmuller ................. 